\title{
Construcción y valoración de las propiedades psicométricas del Cuestionario de Expectativas hacia el Alcohol para Niños de Argentina (CEA-N) \\ Development and psychometric assessment of the Alcohol Expectancy Questionnaire for Argentinean Children (CEA-N)
}

Angelina Pilatti; Juan Carlos Godoy;
Silvina Alejandra Brussino
| Facultad de Psicología. Universidad Nacional de Córdoba.

Enviar correspondencia a:

Angelina Pilatti. Laboratorio de Psicología. Facultad de Psicología. Universidad Nacional de Córdoba. Enrique Barros y Enfermera Gordillo

s/n. Ciudad Universitaria. Córdoba (5000). Córdoba, Argentina.

E-mail: angepilatti@gmail.com.

\section{RESUMEN}

Se construyó y validó un nuevo cuestionario de expectativas positivas y negativas hacia el alcohol para niños de 8 a 12 años. Estudio 1: 117 niños completaron una pregunta estructurada de final abierto sobre los efectos que anticipan del consumo de alcohol. Los registros obtenidos fueron analizados por tres jueces en función de su correspondencia a dos modelos teóricos y a su claridad semántica. Tras analizar el acuerdo entre jueces se obtuvo un conjunto de 55 ítems. Estudio 2: 209 niños completaron el Cuestionario de Expectativas hacia el Alcohol para Niños (CEA-N). El análisis de la estructura interna mediante un análisis factorial exploratorio utilizando el método de componentes principales con rotación oblicua mostró una solución de 5 factores. El análisis de fiabilidad mostró buen valor de confiabilidad para la escala global y la escala riesgo y agresividad y valores moderados para las escalas sociabilidad, relajación, coraje y estados negativos. Estos resultados indican que el CEA-N es un instrumento válido y confiable para la medición de las expectativas hacia el alcohol en niños argentinos.

Palabras clave: expectativas hacia el alcohol, cuestionario, validación psicométrica, 8 a 12 años.
Key words: alcohol expectancies, questionnaire, psychometric assessment; 8 to 12 -year-olds. 


\section{INTRODUCCIÓN}

E inicio temprano del consumo de alcohol representa un importante factor de riesgo para el desarrollo de patrones de consumo abusivo de alcohol (Lo, 2000; Pitkänen, Lyyra y Pulkkinen, 2005; Spear, 2000; Warner y White, 2003). Según estudios realizados en otros países, el consumo de alcohol entre los niños es en mayor parte de tipo experimental y los casos de consumo regular se registran con baja frecuencia (Johnson, Greenlund, Webber y Berenson, 1997). Sin embargo, algunos autores señalan la dificultad de saber cuantos niños han tenido experiencias directas de consumo de alcohol, ya que son muy pocas las encuestas destinadas a evaluar patrones de uso de alcohol en niños en edad escolar (Donovan, Leech, Zucker, Loveland-Cherry, Jester, Fitzgerald et al., 2004). Estudios realizados por nuestro equipo con niños de 8 a 12 años de nuestro medio señalan que la mayoría de los niños (72\%) probó bebidas alcohólicas antes de los 12 años y casi la mitad de éstos últimos respondió consumir bebidas alcohólicas desde algunas veces por año hasta dos 0 tres veces por mes (Pilatti, Godoy y Brussino, 2010).

En las últimas décadas ha surgido una línea de investigación centrada en el concepto de expectativas hacia el alcohol (EA de aquí en adelante). De acuerdo con la definición clásica (Goldman, Brown, Christiansen y Smith, 1991), las EA son las creencias acerca de los efectos que el alcohol produce sobre el comportamiento, el estado de ánimo y las emociones. La percepción de contingencia entre un comportamiento dado y ciertos resultados conduce al almacenamiento de tales asociaciones en la memoria, en la forma de expectativas del tipo "si...entonces" entre el comportamiento y los resultados. Estas asociaciones almacenadas, influyen tiempo después en la decisión de realizar o no un comportamiento dado, en este caso, consumir o no alcohol. Estos efectos anticipados del consumo de alcohol se dividen de manera general como positivos o negativos (mejora en la interacción social y aumento de comportamientos agresivos, respectivamente). Además, y de acuerdo a los efectos farmacológicos observados, otra dimensión considera a las expectativas divididas en expectativas de activación y expectativas de sedación (Goldman y Darkes, 2004). La decisión que lleva a una persona a consumir alcohol estaría guiada en parte por la creencia que el alcohol resultará en consecuencias deseables, como el alivio de la tensión o un mejoramiento del estado de ánimo (D’Amico y Fromme, 2001; Mackintosh, Earleywine y Dunn, 2006; Randolph, Gerend y Miller, 2006; Zamboanga, 2005).

Estas creencias están presentes en los niños antes que tengan su primera experiencia directa con el alcohol (Cameron, Stritzke y Durkin, 2003; Dunn y Goldman, 1996, 1998, 2000; Hipwell, White, Loeber, Stouthamer-Loeber, Chung y Sembower, 2005). Diversos trabajos señalan que los niños más pequeños presentan más EA negativas que los más grandes y una vez que los niños comienzan a experimentar con el alcohol al inicio de la pubertad, las EA se convierten mayormente en expectativas positivas permitiendo predecir el comienzo de la conducta del beber (Catanzaro y Laurent, 2004; Dunn y Goldman, 1998; 2000). Este incremento de las EA positivas junto a la disminución de las EA negativas en función del aumento de la edad ha sido observado en diferentes estudios (Cameron et al., 2003; Dunn y Goldman, 1996; 1998; 2000; Hipwell et al., 2005; Wiers, Sergeant y Boudewijn Gunning, 2000; Query, Rosenberg y Tisak, 1998). Por otra parte, los resultados encontrados en poblaciones adolescentes muestran una asociación entre las expectativas positivas y mayores patrones de consumo de alcohol (D’Amico y Fromme, 2001; Mackintosh et al., 2006; Randolph et al., 2006; Zamboanga, 2005)

La medición de las expectativas hacia el alcohol entre los niños pequeños es más compleja que entre la población adolescente y adulta, ya que hay factores como el nivel de comprensión, el vocabulario y el mantenimiento de la atención, entre otros, que deben ser considerados a la hora del diseño y de la aplicación de los cuestionarios. Entre los cuestionarios desarrollados para evaluar las EA en población infantil figura el Children's Alcohol-Related Expectancies (CARE; Miller, Smith y Goldman, 1990). El CARE es un instrumento de 39 ítems que valora expectativas positivas sobre los efectos que los niños esperan del consumo de alcohol entre los adultos, destinado a niños de nivel inicial (tercer a quinto grado). Para su construcción, los autores modificaron los 55 items con más altas correlaciones item-total de las siete dimensiones del Alcohol Expectancy Questionnaire - Adolescent Form (AEQ-A): cambios globales positivos, cambios en el comportamiento social, mejora en el funcionamiento cognitivo y conductual, incremento de la sexualidad, deterioro cognitivo y conductual, incremento de la activación y relajación. Para disminuir errores relacionados con el nivel de lectura, utilizaron un lenguaje más simple en la redacción de los items y emplearon títeres para administrar el cuestionario. Sin embargo, el uso de títeres fue criticado en base a resultados que mostraron un aumento en las expectativas positivas hacia el alcohol como consecuencia de la exposición a esta metodología (Wiers et al., 2000). Teniendo en cuenta la relación que existe entre el desarrollo de las expectativas positivas y el consumo de alcohol, algunos autores sugieren que debe evitarse cualquier procedimiento que influya positivamente sobre su desarrollo (Wiers et al., 2000). Otra crítica a este instrumento radica en que no incluye expectativas negativas hacia el alcohol y de esta forma no permite obtener una mejor cobertura del tipo de efectos que los niños anticipan del alcohol (Wiers et al., 2000).

Estos mismos autores (Wiers et al., 2000) desarrollaron el Questionnaire for Expectancies in Dutch-Children (QED-C). Este instrumento de 23 ítems, construido a partir de versiones desarrolladas para adolescentes y adultos, mide creencias positivas y negativas sobre los efectos que los niños esperan del consumo de alcohol en las personas, puede ser utilizado con niños de nivel inicial (segundo a quinto grado). Mediante un análisis factorial confirmatorio encontraron un modelo de cuatro factores (mejoramiento sexual, excitación, comportamiento antisocial y ánimo negativo).

El Memory Model-Based Expectancy Questionnaire (MMBEQ) es un instrumento de 41 items (palabras o frases cortas) construido para evaluar las expectativas positivas y negativas de los niños. Del total de ítems, 33 surgieron de discusiones con muestras de niños de segundo a quinto 
grado (Dunn y Goldman, 1996) y 8 ítems de una entrevista realizada a otra muestra de niños. Este instrumento fue utilizado en varias investigaciones para demostrar diferencias en la organización y activación de las expectativas hacia el alcohol en la memoria en función de la edad (Dunn y Goldman, 1998), el consumo (Dunn y Goldman, 1998) y la exposición a comerciales de bebidas alcohólicas (Dunn y Yñiguez, 1999), entre otros. Parte de este instrumento fue utilizado en otros estudios (Cameron et al., 2003; Hipwell et al., 2005) en donde los autores seleccionaron sólo un conjunto del total de ítems.

El interés por el estudio de las expectativas hacia el alcohol se refleja en el crecimiento que esta temática ha tenido desde sus comienzos en la década de los años setenta hasta la actualidad (Pilatti, Godoy y Brussino, 2007). Sin embargo, en nuestro medio son escasas las investigaciones realizadas en esta área. Al respecto, cabe mencionar los estudios realizados por nuestro grupo, cuyos resultados confirmaron la relación existente entre las expectativas hacia el alcohol y los patrones de consumo del mismo en estudiantes universitarios (Cassola, Pilatti y Godoy, 2003) y en adolescentes de colegios de nivel medio (Cassola, Pilatti, Alderete y Godoy, 2005). Por otro lado, en nuestro medio no disponemos de versiones locales de instrumentos diseñados para la medición de las EA. Tenemos conocimiento de la adaptación al español de dos instrumentos construidos en inglés: el Alcohol Expectancy Questionnaire (AEQ; Herrán y Ardila, 2007; Mora-Ríos, Natera, Villatoro y Villalvalzo, 2000) y el Comprehensive Effects of Alcohol (CEOA; Perez-Aranibar, Van den Broucke y Fontaine, 2005; Pilatti y Cassola, 2005). Algunas de estas adaptaciones, si bien son instrumentos fiables y coherentes con los modelos teóricos, no lograron obtener evidencia de las dimensiones propuestas por los modelos de referencia (Perez-Aranibar et al., 2005; Pilatti y Cassola, 2005; Pilatti, Cassola, Godoy y Brussino, 2005). Esto puede deberse al desafío que presenta lograr capturar el exacto significado de algunas palabras que presentan diferencias muy sutiles en relación a la adaptación de otro idioma (Bedregal, Sobell, Sobell y Simco, 2006). Si las expectativas hacia el alcohol están determinadas, al menos en parte, por factores culturales y sociales de los grupos estudiados, la falta de replicabilidad de los modelos teóricos puede deberse a la imposibilidad de las adaptaciones de reflejar variaciones entre una cultura y otra (Herrán y Ardila, 2007; Pérez-Aranibar et al., 2005) o ser reflejo de dificultades metodológicas y conceptuales de los modelos teóricos (Pérez-Aranibar et al., 2005). En este contexto, teniendo en cuenta, por un lado, la relevancia de los aspectos socio culturales tanto en la conducta de consumo como en los efectos que se anticipan de la misma y, por el otro, los antecedentes de adaptaciones locales, se decidió construir un instrumento partiendo de recolectar información local acerca de las creencias de los niños sobre los efectos del alcohol. Para esto, el presente estudio tiene como objetivo desarrollar y validar un instrumento que permita medir las expectativas hacia el alcohol mantenidas por los niños de nuestro medio.

\section{MÉTODO}

\section{Participantes}

Participaron niños con edades comprendidas entre los 8 y 12 años asistentes a colegios de nivel inicial, públicos y privados de las ciudades de Córdoba y Esperanza, provincia de Córdoba y de Santa Fe, respectivamente. La elección de las localidades obedeció a un criterio de tipo accidental. Para la conformación de la muestra, se invitó a colegios del nivel inicial a participar del estudio. La posibilidad de recibir la invitación estuvo determinada por la accesibilidad y disponibilidad de contactos. Entre aquellos colegios que expresaron su intención de colaborar con el estudio (siete instituciones) y de acuerdo a los intereses y posibilidades que presentaron los directivos y docentes, se seleccionaron los cursos que participaron en cada una de las etapas de la investigación. Posteriormente, el investigador principal contactó con los padres, madres o tutores de los niños notificándoles los motivos, la importancia y las consecuencias de la participación de los menores en el estudio. Quedaron excluidos aquellos niños cuyos padres o tutores no dieron su consentimiento para que participasen. Ninguno de los participantes fue incluido en la realización de las dos fases que comprende el presente trabajo.

En la fase de generación de los ítems (fase 1) participaron 117 niños (46\% mujeres). En la fase de administración de los instrumentos (fase 2) participaron 209 niños (52\% mujeres). La distribución de los participantes en función de las variables edad y sexo se observa en la tabla 1.

\section{Procedimiento}

Para que las respuestas de los niños y niñas acerca del uso de alcohol y sus efectos sean confiables, es necesario que reconozcan las bebidas sobre las cuáles se les está preguntando (Donovan et al., 2004). Para evaluar la capacidad de los niños para diferenciar entre bebidas con y sin alcohol y asegurarse que los niños entienden acerca de que se les

Tabla 1: Distribución de los participantes en cada estudio en función de la edad y el sexo

\begin{tabular}{|c|c|c|c|c|c|c|c|c|c|c|c|c|c|c|}
\hline & \multicolumn{2}{|c|}{8 años } & \multicolumn{2}{|c|}{9 años } & \multicolumn{2}{|c|}{10 años } & \multicolumn{2}{|c|}{11 años } & \multicolumn{2}{|c|}{12 años } & \multicolumn{2}{|c|}{ mujeres } & \multicolumn{2}{|c|}{ varones } \\
\hline & $n$ & $\%$ & $n$ & $\%$ & $n$ & $\%$ & $n$ & $\%$ & $n$ & $\%$ & $n$ & $\%$ & $n$ & $\%$ \\
\hline Estudio 1 & 7 & $7 \%$ & 22 & $19 \%$ & 28 & $24 \%$ & 29 & $25 \%$ & 30 & $26 \%$ & 53 & $46 \%$ & 64 & $54 \%$ \\
\hline Estudio 2 & 26 & $12 \%$ & 22 & $11 \%$ & 49 & $23 \%$ & 78 & $37 \%$ & 34 & $16 \%$ & 108 & $52 \%$ & 101 & $48 \%$ \\
\hline
\end{tabular}


está preguntando, se empleó una pregunta de reconocimiento. De esta forma, a cada niño de 8 a 10 años se le mostró una lámina con igual número de figuras de bebidas con alcohol (latas, botellas y vasos de cerveza, vasos y botellas de vino y botellas de fernet, whisky y vodka) y sin alcohol (latas, botellas y vasos de gaseosas y jugos de frutas). Se pidió a los niños que señalasen en la lámina las bebidas alcohólicas. Ningún niño tuvo dificultad para realizar esta tarea y aunque no se les pidió que identificasen un número determinado, todos reconocieron un mínimo de tres bebidas alcohólicas. A los niños de 11 y 12 años se les pidió que mencionasen verbalmente y de manera grupal diferentes tipos de bebidas alcohólicas que ellos conocieran. En ningún caso se mencionaron bebidas no alcohólicas y el investigador a cargo esperó hasta que un ejemplo de cada tipo de bebida alcohólica fuera mencionado. Para estimar las variables referidas a las características sociodemográficas se registró el sexo, la edad, el nivel de estudios y el lugar de residencia de los niños.

En la fase 1 y con el objetivo de obtener material para la construcción del instrumento, se utilizó una pregunta estructurada de final abierto. Para evitar errores producto de la dispersión o a dificultades de lectura y/o comprensión, con los niños de 8 a 10 años se trabajó de manera individual en el espacio cedido por cada colegio. El investigador a cargo leyó a cada participante las instrucciones y las preguntas, registrando por escrito las respuestas dadas verbalmente. Con los niños de 11 y 12 años la administración fue colectiva y tuvo lugar en el aula de clases. Si bien las instrucciones y las preguntas fueron leídas por el investigador, los participantes registraron sus respuestas en sus hojas. En este caso, el cuestionario de pregunta abierta se completó dentro de un mismo día en cada escuela para minimizar la comunicación entre los participantes, controlando así una fuente potencial de contaminación (Dunn y Goldman, 2000). Si bien con los niños de 8 a 10 años la administración individual ocupó más de un día por escuela, los participantes de un mismo grado completaron las preguntas durante un mismo día. En ningún caso se solicitó información referida a la identidad de los participantes. Antes de comenzar, se enfatizó la naturaleza voluntaria de la participación y se explicó que el interés del estudio era conocer lo que ellos pensaban acerca de los efectos del alcohol en el comportamiento y las emociones de las personas. Si bien los niños no firmaron una nota de consentimiento informado, el investigador principal señaló a los participantes que podian abandonar el estudio en cualquier momento si así lo querían. La recolección de datos estuvo a cargo del investigador principal.

Fase 1: Construcción de los items: dos semanas antes de la recolección de datos, el investigador principal se presentó a los niños explicando la realización del estudio. Se entregaron las notas de información y autorización para que los niños llevaran a sus hogares. Dos semanas más tarde, el investigador principal regresó a la escuela y a los niños autorizados por sus padres a participar se les administró el cuestionario abierto de expectativas hacia el alcohol para la obtención de la información necesaria para la construcción de los ítems referidos a los efectos que los niños anticipan del consumo de alcohol. Para esto, todos los niños respon- dieron a la siguiente pregunta abierta: "¿cómo se sienten las personas cuando toman bebidas alcohólicas?" (Dunn y Goldman, 2000). Se utilizó el genérico personas como grupo de referencia ya que entre los niños más pequeños la experiencia directa de consumo es baja (Donovan et al., 2004). Se les pidió a los niños que "respondieran lo más rápido que pudieran, sin detenerse a pensar mucho y utilizando palabras o frases cortas". De este modo se buscaba medir de un modo más directo los contenidos almacenados en la memoria en relación a conceptos específicos ya que se considera que las respuestas dadas por los participantes corresponden a las asociaciones almacenadas en la memoria en relación al estímulo alcohol. Responder rápidamente tiene el objetivo de reducir la intervención de otros pensamientos que puedan contaminar la respuesta (Nelson, McEvoy y Dennos, 2000).

Una vez obtenidos los registros, se convocó a un grupo de tres jueces expertos en el área de construcción de pruebas o del uso y abuso de alcohol para realizar la evaluación semántica y el análisis de la concordancia de cada uno de los registros obtenidos. Cada juez recibió la definición conceptual de la variable expectativas hacia el alcohol y dos sistemas de categorización correspondientes a dos modelos teóricos: el de Fromme, Stroot y Kaplan (1993) y el de Dunn y Goldman (2000).

El modelo de Fromme et al., (1993) divide las expectativas hacia el alcohol en función de la valencia del efecto esperado (positivas vs. negativas) y especificamente de acuerdo a la dimensión de la conducta a la que refieren las consecuencias anticipadas del consumo (sociabilidad, relajación, coraje y deterioro cognitivo y conductual, riesgo y agresividad y percepción de estados negativos). Si bien este modelo considera además la dimensión "sexualidad", ésta no fue incluida en el sistema de categorización, ya que los registros obtenidos no incluian expectativas referidas a la interacción sexual.

Por otra parte, el modelo de Dunn y Goldman (2000) considera a las expectativas en función de dos aspectos principales: valencia (con los extremos positivo y negativo) y activación (con los extremos activación y sedación). Específicamente, este modelo plantea cuatro factores que corresponden a los cuatro cuadrantes que se observan mediante escalamiento multidimensional: cambios sociales positivos (creencias relacionadas con la activación de emociones positivas y con un incremento en la interacción social), factor de activación negativa (incluye la activación de emociones y conductas negativas como lastimar a otros, estar triste), factor deterioro/sedación (contiene creencias acerca de un deterioro cognitivo y conductual) y factor de desenfreno (incluye expectativas sobre la presencia de emociones y conductas alocadas y desenfrenadas como consecuencia del alcohol).

A cada juez se le pidió que intentase ubicar cada ítem en las dimensiones de la variable expectativas hacia el alcohol de acuerdo a las categorías ofrecidas por cada uno de los dos modelos de categorización y que realizase una evaluación de la calidad de cada ítem considerando los siguientes aspectos: corrección sintáctica, claridad semántica y la adecuación para la población a la cual está dirigido el instrumento. Una puntuación de 10 indicaba que se trataba de un ítem de alta 
calidad al cumplir con los criterios anteriormente enunciados, mientras que una puntuación de 1 indicaba una baja calidad y la sugerencia implícita de no incluirlo en la construcción de la prueba. Además, se brindó un espacio para que los jueces realizaran aquellas observaciones que consideraran pertinentes o anexaran información que pudiera ser relevante para la investigación. Teniendo en cuenta que estas clasificaciones corresponden a instrumentos desarrollados en otros países, con otros ítems y para población adolescente, se pidió a los jueces que incorporasen nuevas categorías en caso de ser necesario para una correcta clasificación de los ítems.

Fase 1: Instrumento final: Mediante el procedimiento detallado en la sección anterior se obtuvieron 75 palabras o frases cortas. Con relación a los modelos de clasificación de los ítems, se encontró que el modelo de Fromme et al. (1993) presentó mayor acuerdo entre los jueces. En base a esto, se calculó el porcentaje de acuerdo entre los jueces al clasificar cada uno de los 75 registros según este modelo. Se retuvieron los ítems con un 66\% o más de acuerdo entre los jueces, esto es, donde al menos dos jueces coincidieran en la categorización (Rather, Goldman, Roehrich y Brannick, 1992). Siguiendo este criterio se encontró un ítem en donde no hubo acuerdo (el alcohol hace que las personas se pongan nerviosas), tras cuya eliminación se obtuvo un grupo de 74 ítems. De acuerdo con las sugerencias realizadas, se volvieron a redactar algunos ítems utilizando palabras más simples y desdoblando ítems multidimensionales. Luego se agruparon aquellos de significado similar, manteniendo el ítem con mayor puntuación en la evaluación de su claridad semántica. Los ítems finales fueron aplicados a una muestra de diez niños en una prueba piloto sin que se presentasen dudas o preguntas acerca de su significado. Se obtuvo un cuestionario de 55 ítems, distribuidos en seis escalas de la siguiente forma en función de la clasificación realizada por los jueces: incremento de la sociabilidad: mejora en el desempeño social (10 ítems), relajación y reducción de la tensión (9 ítems), coraje: sentimientos de seguridad y confianza (10 ítems), deterioro cognitivo y conductual (8 ítems), riesgo y agresión: aumento de la agresividad y toma de riesgos (10 ítems) y percepción de estados negativos (8 ítems).

Los efectos del alcohol en el área sociabilidad se relacionan con un aumento de la interacción social general (poder hablar más, ser más amigables) y con la posibilidad de divertirse y pasarlo bien (divertirse en fiestas, contar chistes, ser divertido). Respecto a la escala relajación y reducción de la tensión, hace referencia al reforzamiento positivo (aumento de la relajación: pasarla mejor, estar cómodo, sentirse contento, estar de buen humor) y al reforzamiento negativo o cualidad ansiolítica (disminución o eliminación de un estímulo aversivo: preocuparse menos por problemas, tranquilizarse si estaba nervioso) del alcohol. Una diferencia encontrada con el ítem estar de buen humor, es que en el modelo de referencia se ubica en la escala sociabilidad y en el presente trabajo en la escala relajación. Esto puede deberse a que en nuestro medio estar de buen humor puede relacionarse más con la posibilidad de estar tranquilo y sin preocuparse por problemas que con la posibilidad de interactuar con las personas. En la escala coraje se distinguen dos grupos de efec- tos, uno que describe un efecto general de superioridad (más fuertes, más que los otros) y otro la valentía para expresarse libremente (decir lo que sienten, lo que piensan, animarse a hacer cosas, seguridad en uno mismo). Con relación a las expectativas negativas, los ítems de la escala riesgo y agresividad refieren exclusivamente a conductas agresivas hacia los demás (lastimar, ofender, molestar, discutir, maltratar), sin contemplar aspectos referidos a la toma de riesgos. La escala percepción de estados negativos contempla efectos negativos del alcohol a más largo plazo, ya que incluye principalmente sentimientos de culpa, vergüenza y arrepentimiento por lo que se hizo y dijo. La escala deterioro cognitivo y conductual cubre aspectos negativos a corto plazo, relacionados con una disminución del rendimiento y un entorpecimiento conductual y físico general (descompuestas, dolor de cabeza, mareadas, con pérdida del equilibrio).

Fase 2: Administración del instrumento: una vez finalizada la fase correspondiente a la construcción de los ítems, al igual que en la fase anterior, el investigador principal se presentó en los colegios para informar a los niños acerca de la investigación que se llevaría a cabo y para repartir las notas de autorización para que los niños llevaran a sus hogares. Dos semanas después, el investigador principal volvió al colegio donde se realizó la administración del cuestionario de 55 ítems obtenido en la fase 1. La administración individual implicó un tiempo de 25 minutos y la administración colectiva 40 minutos.

\section{Instrumentos}

Cuestionario de Expectativas hacia el Alcohol para Niños (CEA-N): de acuerdo a los resultados de la fase 1, este instrumento está conformado por 55 ítems agrupados en seis escalas que valoran los efectos positivos (incremento de la sociabilidad, relajación y reducción de la tensión, coraje: sentimientos de seguridad y confianza) y negativos (deterioro cognitivo y conductual, riesgo y agresividad, percepción de estados negativos) que los niños anticipan como consecuencia del consumo de alcohol de las personas. Para completar el cuestionario, cada niño debía responder en una escala de cuatro puntos (nunca, pocas veces, muchas veces, siempre) cuántas veces después de tomar alcohol las personas se sentían del modo que describía cada ítem. Siguiendo parte de la técnica empleada por Dunn y Goldman (1996), con los niños de 8 a 10 años se utilizó una plantilla con cuatro rectángulos con diferentes superficies sombreadas, cada uno equivalente a una de las opciones de respuesta. Luego de que el entrevistador leía cada ítem, el niño debía señalar en la plantilla el rectángulo correspondiente a su respuesta. La opción de respuesta nunca estaba representada por un rectángulo blanco, pocas veces por un rectángulo parcialmente sombreado, muchas veces por dos tercios de la figura sombreados y siempre por el rectángulo completamente sombreado. Los niños de 11 y 12 años completaron el cuestionario encerrando el casillero correspondiente a su respuesta. En este caso, cada opción de respuesta estaba sombreada con una intensidad que iba en aumento en función de la frecuencia que indicaba. De esta forma, el casillero de la opción nunca estaba 
en blanco, el correspondiente a pocas veces, sombreado de modo ligero con color gris y los casilleros de muchas veces y siempre cada uno con una tonalidad más oscura de color gris que el anterior. En los dos casos, se buscó hacer una analogía entre una mayor superficie (niños de 8 a 10 años) o mayor intensidad de sombreado (niños de 11 y 12 años) y la una mayor cantidad de veces en que se anticipaba ese efecto.

\section{RESULTADOS}

\section{Análisis de Datos}

Para evaluar la evidencia de estructura del CEA-N y la fiabilidad de la misma se realizó un análisis factorial exploratorio y se estimó el coeficiente alfa de Cronbach (para la prueba global y para cada escala y eliminando uno a uno cada ítem), respectivamente.

\section{Preparación de los datos}

Como primera medida se realizó una exploración inicial de los datos, cumpliendo de este modo con un requisito básico al utilizar técnicas multivariadas (Hair, Anderson, Tatham y Black, 1999). En primer lugar se evaluó el patrón de valores perdidos para estimar si el mismo respondía a una distribución aleatoria y para evaluar el porcentaje de estos valores en cada variable. Mediante la rutina de Análisis de los Valores Perdidos del SPSS 15 no se observaron porcentajes de datos ausentes mayores al 5\% en ninguno de los 55 ítems. Teniendo en cuenta este bajo porcentaje de datos perdidos, se decidió reemplazarlos mediante el método de imputación de sustitución por la media de la cada variable. Luego, se identificaron casos atípicos univariados mediante el cálculo de puntuaciones estándar para cada una de las variables. Se consideraron atípicos aquellos casos con puntuaciones $z$ superiores a 3.29 (prueba de dos colas, $p<.001$ ) y que aparecieran identificados como tales en los gráficos de cajas (Boxplots). Siguiendo este criterio, no se encontraron casos que presentaran patrones de respuesta atípicos. Finalmente, para comprobar los supuestos de normalidad de la muestra se realizaron análisis de asimetría y curtosis en cada variable y una inspección grafica de la distribución de los puntajes (histogramas con curva normal). Se observó que 41 items presentaron índices de curtosis y simetría entre +1.00 y -1.00 , considerado por la literatura como excelente y 14 items presentaron índices de asimetría y curtosis inferiores a 1.60, considerado adecuado (George y Mallery, 2003).

\section{Análisis Factorial}

Para analizar la dimensionalización de los 55 ítems del CEA-N se realizó un análisis factorial exploratorio empleando el método de los componentes principales. La factibilidad del análisis se evaluó a través del índice de adecuación muestral KMO (Kaiser-Meyer-Olkin) que presentó un valor de .753 y la prueba de esfericidad de Bartlett que presentó resultados significativos $(\mathrm{df}=1485 ; \mathrm{p}<.001)$. Para la determinación del número de factores se utilizó en una primera instancia la regla de Kaiser-Gutman que consiste en retener aquellos factores con autovalores mayores a 1. Siguiendo este procedimiento se obtuvo una solución de 16 factores que explicaban un $63,39 \%$ de la varianza. Sin embargo, el uso de la regla de Kaiser-Gutman ha sido cuestionado por algunos autores por retener un número elevado de factores y por considerarse como un método poco preciso (Costello y Osborne, 2005; Velicer y Jackson, 1990).

Por medio de la inspección se analizaron las cargas factoriales de los ítems, considerando como criterios de retención que presentasen una carga factorial mayor a .40 y que mantuviesen correspondencia con la clasificación realizada por los jueces. Mediante este procedimiento, se encontró que los ítems pertenecientes a la escala deterioro cognitivo y conductual presentaban un comportamiento irregular, en tanto que correlacionaron de manera aislada en diferentes factores, sin que se encontrase una agrupación clara entre ellos. Este resultado es similar al presentado en otros trabajos en donde también se registraron dificultades con los items de esta escala (Miller et al., 1990; Wiers et al., 2000). Siguiendo los criterios de retención mencionados se obtuvo un conjunto de 28 ítems. Luego se agregaron dos ítems que si bien no presentaban correspondencia con la clasificación de los jueces, mantenían relevancia teórica con el resto de los elementos de los factores. Específicamente, el ítem reirse mucho perteneciente a la escala relajación se correlacionó con los ítems de la escala sociabilidad y el ítem sean alegres perteneciente a la escala sociabilidad se agrupó junto a los items de la escala relajación.

Se analizó la estructura factorial de estos 30 ítems mediante rotación oblicua. Si bien las rotaciones ortogonales, como la varimax, son las más empleadas al realizar un análisis factorial en tanto que producen una solución de factores no relacionados (Costello y Osborne, 2005; Hair et al., 1999; Johnson, 2000), en las ciencias sociales en general (Costello y Osborne, 2005) y en el campo de las expectativas hacia el alcohol en particular (Goldman et al., 1991) se espera un grado de asociación entre los factores que requiere el empleo de una rotación oblicua. La factibilidad del análisis se determinó a través de los mismos indicadores mencionados anteriormente (índice de adecuación muestral $\mathrm{KMO}=$ .792; prueba de esfericidad de Bartlett con resultados significativos: $d f=435 ; p<.001)$. De acuerdo a la interpretación del gráfico de sedimentación se determinó la extracción de 5 factores que en conjunto explicaron el 48,72\% de la varianza. El primer factor denominado riesgo y agresividad (9 ítems) explicó el 15,17\% de la varianza total y el segundo factor, identificado como incremento de la sociabilidad (7 items), el $14,9 \%$ de la varianza. El tercer ( 5 ítems), cuarto (4 ítems) y quinto factor (5 ítems), denominados percepción de estados negativos, coraje: sentimientos de seguridad y confianza y relajación y reducción de la tensión, respectivamente, explicaron el $8,56 \%, 5,36 \%$ y $4,41 \%$ de la varianza. Las cargas factoriales obtenidas se presentan en la tabla 2. 


\section{Consistencia interna}

Los resultados encontrados al analizar la fiabilidad del instrumento mediante el cálculo del coeficiente alfa de Cronbach señalan que la escala global y una de las cinco escalas (riesgo y agresividad) presentan altos valores de fiabilidad ( $\alpha=.80$ y $\alpha=.83$, respectivamente). En las cuatro escalas restantes se observaron valores moderados, pero adecuados de consistencia interna (sociabilidad: $\alpha=.74$; relajación: $\alpha=.75$, percepción estados negativos: $\alpha=.75 \mathrm{y}$ coraje: $\alpha=.71$ ).
Al analizar la fiabilidad de cada escala eliminando uno a uno los ítems que la componen, se confirmó la decisión de incluir el ítem reírse mucho en la escala sociabilidad y el ítem sean alegres en la escala relajación. En ambos casos, la eliminación de estos ítems se tradujo en una marcada disminución del índice de fiabilidad de las respectivas escalas. Al analizar el aporte de cada ítem a la fiabilidad de su correspondiente escala, en ningún caso se observó que la eliminación de un item se traduzca en un aumento de la fiabilidad. Los valores del coeficiente alfa para cada escala eliminando uno a uno cada ítem se presentan en la tabla 2.

Tabla 2: Cargas factoriales y coeficiente alfa de Cronbach al eliminar ese ítem de los 30 ítems finales del CEA-N

\begin{tabular}{|c|c|c|c|c|c|c|c|}
\hline & $\mathrm{F} 1^{*}$ & $\mathrm{~F} 2^{*}$ & $\mathrm{~F} 3^{*}$ & $\mathrm{~F} 4^{*}$ & $\mathrm{~F}^{*}$ & $\begin{array}{c}\alpha \sin \text { el } \\
\text { item }\end{array}$ & c \\
\hline \multicolumn{8}{|l|}{ "El alcohol hace que las personas..." } \\
\hline Busquen pelearse con otras personas &, 568 & & & & & .825 & \\
\hline Digan malas palabras & 658 & & & & & .812 & \\
\hline Sean agresivas &, 736 & & & & & .803 & \\
\hline Lastimen a otras personas & 697 & & & & & .809 & \\
\hline Ofendan a los demás &, 623 & & & & & .813 & \\
\hline Traten mal a otras personas &, 706 & & & & & .803 & \\
\hline Se enojen con facilidad &, 495 & & & & & .820 & \\
\hline Molesten a los demás &, 622 & & & & & .807 & \\
\hline Discutan con los demás & 660 & & & & & .807 & \\
\hline Escala "riesgo y agresividad" & & & & & & & .83 \\
\hline Hagan cosas que hacen reír a los demás & & 689 & & & & .712 & \\
\hline Cuenten chistes & & 644 & & & & .700 & \\
\hline Sean más simpáticas & &, 504 & & & & .719 & \\
\hline Sean divertidas & &, 710 & & & & .696 & \\
\hline Se diviertan más en una fiesta & & 481 & & & & .709 & \\
\hline Se rian mucho & &, 682 & & & & .686 & \\
\hline Hablen más & &, 527 & & & & .717 & \\
\hline Escala "sociabilidad" & & & & & & & .74 \\
\hline Digan cosas de las que después se arrepienten & & &, 741 & & & .694 & \\
\hline Se sientan tristes & & &, 551 & & & .748 & \\
\hline Sientan vergüenza por lo que hacen cuando toman & & &, 716 & & & .700 & \\
\hline Se sientan culpables por las cosas que dicen... & & &, 776 & & & .683 & \\
\hline Se sientan culpables por las cosas que hacen... & & &, 708 & & & .706 & \\
\hline Escala "estados negativos" & & & & & & & .75 \\
\hline Piensen que son más que los demás & & & &,- 672 & & .654 & \\
\hline Se sientan superiores al resto & & & &,- 720 & & .619 & \\
\hline Se sientan más lindas que los demás** & & & &,- 734 & & .650 & \\
\hline Se sientan más fuertes que los demás & & & &,- 652 & & .641 & \\
\hline Escala "coraje y seguridad" & & & & & & & .71 \\
\hline Se sientan contentas & & & & &, 630 & .670 & \\
\hline Estén de buen humor & & & & &, 575 & .722 & \\
\hline Sean alegres & & & & &, 642 & .685 & \\
\hline Se sientan cómodas con lo que están haciendo & & & & &, 574 & .733 & \\
\hline La pasen mejor en lo que estén haciendo & & & & & 689 & .702 & \\
\hline Escala "relajación" & & & & & & & .75 \\
\hline
\end{tabular}

*F1: primer factor; F2: segundo factor; F3: tercer factor; F4: cuarto factor; F5: quinto factor

** (Que las personas se sientan más lindas significa que las personas se sienten más bonitas) 


\section{DISCUSIÓN}

En el presente trabajo se presentan los procedimientos realizados en la construcción y valoración de las propiedades psicométricas del Cuestionario de Expectativas hacia el Alcohol para Niños (CEA-N). De acuerdo a los resultados obtenidos, se apoya el uso del CEA-N para valorar el tipo de EA mantenidas por los niños de nuestro medio. Dos aspectos fundamentales destacan la relevancia y el aporte de este estudio.

En primer lugar, hasta donde tenemos conocimiento, este es el primer instrumento desarrollado y validado en nuestro medio para medir las expectativas hacia el alcohol en niños. Al respecto, la tradición en esta área señala un predominio de adaptaciones de versiones para adolescentes, descuidando diferencias evolutivas que pueden ser sustanciales en una conducta tan compleja como el consumo de alcohol. En base a esto, este trabajo se propuso relevar los efectos que niños y niñas de nuestro medio anticipan como consecuencia del consumo de alcohol, buscando de esta manera, que el instrumento refleje de la manera más real posible las expectativas hacia el alcohol de los niños.

En segundo lugar, el análisis de la estructura interna y de la fiabilidad del CEA-N indica que es un instrumento válido y confiable para valorar los efectos positivos y negativos que los niños anticipan del consumo de alcohol de las personas. Al respecto, la estructura interna encontrada muestra un modelo de cinco factores que explica casi el 50\% de la varianza. El primer factor correspondiente a la dimensión riesgo y agresividad refleja una situación de pérdida de control expresada a través de conductas de maltrato físico y verbal dirigidas hacia los demás, sin incluir aspectos relacionados a otro tipo de comportamientos riesgosos como participar en conductas de riesgo (Fromme et al., 1993) y sentirse alocado y desenfrenado (Dunn y Goldman, 1996). La dimensión incremento de la sociabilidad explica casi el mismo porcentaje de varianza que el primer factor y se relaciona con el papel del alcohol como facilitador de las interacciones sociales. De esta manera, la anticipación de efectos relacionados con el aumento de la agresividad por un lado y con una mejora en la sociabilidad por el otro, realizan un aporte similar en la explicación de la varianza. En el tercer factor se agruparon los ítems de la escala percepción de estados negativos relacionados con la culpa, vergüenza y arrepentimiento ocasionados a partir de las cosas dichas y hechas bajo los efectos del alcohol. En este sentido, es muy marcada la connotación moral y crítica que caracteriza esta dimensión, con una evaluación negativa del comportamiento mantenido bajo los efectos del alcohol. Por otro lado, mientras que los ítems de la escala riesgo y agresividad corresponden a conductas desarrolladas bajo la influencia del alcohol (corto plazo), los items de la escala estados negativos reflejan consecuencias a largo plazo, esto es, tras recuperar el control. Los ítems que componen el cuarto factor corresponden a la anticipación de un incremento de la seguridad y confianza basado en sentimientos de superioridad respecto a los demás (más fuerte, más lindo, más que los otros). La quinta dimensión denominada relajación y disminución de la tensión queda conformada por ítems que describen el efecto del alcohol sólo como reforzador positivo, en tanto que aumenta la sensación de bienestar general (sentirse alegre, contento y cómodo). Al respecto, la falta de ítems en el modelo final que reflejen la anticipación de una disminución o eliminación de estados aversivos puede estar relacionada con la falta de exposición de los niños a este tipo de efectos.

EI CEA-N, a diferencia de otros instrumentos, no contempla las expectativas hacia el alcohol pertenecientes a las dimensiones que en la literatura se denominan incremento de la sexualidad (Fromme et al., 1993; Miller et al., 1990, Wiers et al., 2000) y deterioro cognitivo y conductual (Brown, Christiansen y Goldman, 1987; Fromme et al., 1993). Al respecto, los instrumentos para niños que contienen la escala incremento de la sexualidad (Miller et al., 1990, Wiers et al., 2000) son adaptaciones de versiones desarrolladas para población adolescente, $y$ en este sentido, no necesariamente reflejan el contenido de las creencias mantenidas por los niños. Como ya se mencionó anteriormente, cada uno de los items del CEA-N fue obtenido a partir de una muestra de niños de nuestro medio, por lo cual es esperable que reflejen de un modo más cercano a la realidad el contenido de sus creencias sobre los efectos del alcohol, que en este caso, no incluian elementos pertenecientes a la dimensión incremento de la sexualidad.

La eliminación de la escala deterioro cognitivo y conductual coincide con otros estudios con niños (Miller et al., 1990; Wiers et al., 2000), donde también se descartó esta escala ya que las respuestas a los ítems eran muy sesgadas. En el presente trabajo, en un análisis previo de la consistencia interna (de acuerdo a la clasificación realizada por los jueces) se registraron valores inadecuados para esta escala ( de Cronbach $=.49$ ). Sumado a esto, el análisis de la estructura interna mostró un patrón inconsistente entre estos ítems, sin que se encuentre una agrupación clara entre los mismos. Esto puede deberse a que los niños de esta edad no han desarrollado aún la capacidad de poder reflexionar acerca del funcionamiento cognitivo (Miller et al., 1990), lo cual se traduce en falta de consistencia encontrada.

Otra caracteristica del CEA- $\mathrm{N}$ radica en la especificidad de sus ítems, en tanto que no refieren a efectos generales sino que delimitan un efecto concreto y particular, lo cual hace más fácil y accesible que los niños puedan comprender los items.

Con relación a la consistencia interna, la escala global y la escala riesgo y agresividad presentan buen nivel de fiabilidad, mientras que el resto de las escalas presentan valores moderados, todos dentro de los rangos considerados aceptables. Al respecto, es importante tener en cuenta que la fiabilidad obtenida en trabajos con niños suele ser menor que cuando se trabaja con adolescentes o adultos, debido a que las respuestas de los niños suelen ser más inestables (Lemos, 2006; Smith, 2008). Además, los valores de fiabilidad encontrados en otras investigaciones son similares y aún más bajos que los reportados en este trabajo (Dunn y Goldman, 1996; Hipwell et al., 2005; Kraus, Smith y Ratner, 
1994; Miller et al., 1990; Wiers et al., 2000). Por otro lado, salvo el trabajo de Miller et al. (1990), en la mayoria de estos estudios se reportaron los coeficientes de fiabilidad obtenidos para la escala global (Dunn y Goldman, 1996; Kraus et al., 1994) o en función de agrupar las expectativas hacia el alcohol positivas por un lado y las expectativas hacia el alcohol negativas por el otro (Hipwell et al., 2005; Wiers et al., 2000). En cualquiera de los dos casos, aún cuando el cálculo de la consistencia interna fue realizado considerando un número mayor de ítems que el correspondiente a cada una de las escalas del presente trabajo, los valores obtenidos no son mayores a los aquí reportados.

Si bien las propiedades psicométricas observadas apoyan el uso del CEA-N como una herramienta válida y confiable, es importante mencionar las limitaciones que presenta este trabajo. Por un lado, la muestra de participantes fue seleccionada de modo accidental y en este sentido, los resultados no pueden generalizarse a toda la población. Por otro lado, sería conveniente realizar estudios que permitan confirmar los resultados aquí reportados. Teniendo en cuenta por un lado, la importancia de utilizar una muestra diferente para confirmar los resultados de un análisis exploratorio y por el otro, que el número de casos no era suficiente para dividir la muestra en dos partes (una para el análisis exploratorio y otra para el confirmatorio), se propone realizar en un estudio posterior un análisis factorial confirmatorio que permita verificar la estructura aquí encontrada.

De acuerdo a todo lo expuesto, el CEA-N constituye una herramienta válida y confiable para ser utilizada en el medio local para la medición de las expectativas hacia el alcohol mantenidas por niños de 8 a 12 años. Su uso posibilitará no sólo obtener una explicación más completa y exhaustiva de la conducta de consumo, sino que como herramienta diagnóstica, permitirá además, identificar poblaciones de riesgo con relación a la participación actual o potencial en conductas de abuso de alcohol. Al respecto, la línea de trabajos a realizar para continuar los resultados reportados en el presente trabajo, implica además de la confirmación de la estructura interna aquí reportada, la realización de estudios longitudinales y que permitan delimitar con mayor exactitud el rol de las EA en el inicio y mantenimiento de la conducta de consumo de alcohol entre los niños de nuestro medio.

\section{AGRADECIMIENTOS Y FINANCIAMIENTO}

El presente trabajo ha sido posible gracias a una beca a nombre de Angelina Pilatti del Consejo Nacional de Investigaciones Científicas y Técnicas (CONICET).

El primer autor agradece la colaboración de Luciano F. Ponce en la corrección del texto.

\section{REFERENCIAS}

Bedregal, L., Sobell, L., Sobell, M. y Simco, E. (2006). Psychometric characteristics of a Spanish version of the DAST-10 and the RAGS. Addictive Behaviors, 31, 309-319

Brown, S. A., Christiansen, B. A. y Goldman, M. S. (1987). The Adolescent Expectancy Questionnaire: An instrument for the assessment of adolescent and adult alcohol expectancies. Journal of Studies on Alcohol 48, 483-491.

Cameron, C. A., Stritzke, W. G. y Durkin, K. (2003). Alcohol expectancies in late childhood: An ambivalence perspective on transition toward alcohol use. Journal of Child Psychology and Psychiatry, $44,687-698$.

Cassola, I., Pilatti, A., Alderete, A. y Godoy, J. (2005) Conductas de riesgo, expectativas hacia el alcohol y consumo de alcohol en adolescentes de la ciudad de Córdoba. Revista Electrónica Evaluar, 5, 38-54.

Cassola, I., Pilatti, A. y Godoy, J. (2003). Expectativas hacia el alcohol y consumo de alcohol en estudiantes universitarios. Universidad Nacional de Córdoba.

Catanzaro, S. J. y Laurent, J. (2004). Perceived family support, negative mood regulation expectancies, coping, and adolescent alcohol use: Evidence of mediation and moderation effects. Addictive Behaviors, 1-19.

Costello, A. B. y Osborne, J. W. (2005). Best practices in exploratory factor analysis: four recommendations for getting the most from your analysis. Practical Assessment Research y Evaluation, 10, 1-9

D'Amico, E. y Fromme, K. (2001). Brief intervention for adolescent risk-taking behavior. Addiction, 97, 563-574.

Donovan, J. E., Leech, S. L., Zucker, R. A.; Loveland-Cherry, C. J., Jester, J. M., Fitzgerald, H. E., et al. (2004). Really underage drinkers: Alcohol use among elementary students. Alcoholism: Clinical and Experimental Research, 28, 341-349

Dunn, M. E. y Goldman, M. S. (1996). Empirical modeling of an alcohol expectancy network in elementary school children as a function of grade. Experimental and Clinical Psychology, 4, 209-217.

Dunn, M. E. y Goldman, M. S. (1998). Age and drinking- related differences in the memory organization of the alcohol expectancies in 3rd-, 6th-, 9th-, and 12th-grade children. Journal of Consulting and Clinical Psychology, 66, 579-585.

Dunn, M. E. y Goldman, M. S. (2000). Validation of multidimensional scaling-based modeling of alcohol expectancies in memory: age and drinking-related differences in expectancies of children assessed as first associates. Alcoholism: Clinical and Experimental Research, 24, 1639-1346.

Dunn, M. E. e Yniguez, R. M. (1999). Experimental demonstration of the influence of alcohol advertising on the activation of alcohol expectancies in memory among fourth- and fifth-grade children. Experimental and Clinical Psychology, 7, 473-483.

Fromme, K., Stroot, E. y Kaplan, D. (1993). Comprehensive Effects of Alcohol: Development and psychometric assessment of a new expectancy questionnaire. Psychological Assessment, 5, 19-26.

George, D. y Mallery, M. (2003). Using SPSS for Windows step by step: a simple guide and reference. Boston, MA: Allyn y Bacon.

Goldman, M. S. y Darkes, J. (2004). Alcohol expectancy multiaxial assessment: a memory network- based approach. Psychological Assessment, 16, 4-15. 
Goldman, M. S., Brown, S. A.; Christiansen, B. A. y Smith, G. T. (1991). Alcoholism and memory: Broadening the scope of alcoholexpectancy research. Psychological Bulletin, 110, 137-146

Hair, J. F., Anderson, R. E., Tatham, R. L. y Black, W. C. (1999). Análisis Multivariante. Madrid: Prentice Hall Iberia.

Herrán, O. F. y Ardila, M. F. (2007). Validez factorial del cuestionario de expectativas hacia el consumo de alcohol (AEQ-III), en adultos de Bucaramanga, Colombia. Revista Médica de Chile, 135, 488-495.

Hipwell, A. E., White, H. R., Loeber, R., Stouthamer-Loeber, M., Chung, T. y Sembower, M. A. (2005). Young girls' expectancies about the effects of alcohol, future intentions and patterns of use. Journal of Studies on Alcohol, 6, 630-639

Johnson, C. C., Greenlund, K. J., Webber, L. S. y Berenson, G. S. (1997). Alcohol first use and attitudes among young children. Journal of Child and Family Studies, 6, 359-372

Johnson, D. E. (2000). Métodos multivariados aplicados al análisis de datos. México: Internacional Thomson Editores (trabajo original publicado en 1998).

Kraus, D., Smith, G. T. y Horn Ratner, H. (1994). Modifying alcoholrelated expectancies in grade-school children. Journal of Studies on Alcohol, 55, 535-542

Lemos, V. (2006). La deseabilidad social en la evaluación de la personalidad infantil. Suma Psicológica, 13, 7-14.

Lo, C (2000). Timing of drinking initiation: A trend study predicting drug use among high school seniors. Journal of Drug Issues, 30 , 525-554.

Mackintosh, A. M., Earleywine, M. y Dunn, M. E. (2006). Alcohol expectancies for social facilitation: A short form with decreased bias. Addictive Behaviors, 31, 1536-1546.

Miller, P. M., Smith, G. T. y Goldman M. S. (1990). Emergence of alcohol expectancies in childhood: A possible critical period. Journal of Studies on Alcohol, 51, 343-349.

Mora-Ríos, J., Natera, G., Villatoro, J. y Villalvalzo, R. (2000). Validez factorial del Cuestionario de Expectativas hacia el Alcohol (AEO) en estudiantes universitarios. Psicología Conductual, 8, 319-328.

Nelson, D. L., McEvoy, C. L. y Dennis, S. (2000). What is free association and what does it measure? Memory and Cognition, 28, 887-899.

Perez-Aranibar, C., Van den Broucke, S. y Fontaine, J. (2005). Validation of the Alcohol Expectancy Questionnaire (AEQ-A) for Peruvian University Students. Journal of Alcohol y Drug Education, 49, 63-84.

Pilatti, A. y Cassola, I. (2005). Conductas de riesgo, expectativas hacia el alcohol y consumo de alcohol en adolescentes de poblaciones carenciadas de la ciudad de Córdoba. Secretaria de Extensión Universitaria. Facultad de Psicología. Universidad Nacional de Córdoba.

Pilatti, A., Cassola, I., Godoy, J. y Brussino, S. (2005). Validez factorial del Cuestionario de Expectativas hacia el Alcohol. Las Ciencias del Comportamiento en los Albores del siglo XXI. Universidad Nacional de Mar del Plata.

Pilatti, A., Godoy, J. y Brussino, S. (2010). Expectativas hacia el alcohol y experimentación con el alcohol en niños de 8 a 12 años. Manuscrito en preparación

Pilatti, A., Godoy, J. y Brussino, S. (2007). Análisis bibliométrico sobre la temática: Medición de las expectativas hacia el alcohol. Avances en Investigación en Ciencias del Comportamiento en Argentina. Universidad del Aconcagua. Tomo II: 1069-1094.

Pitkänen, T., Lyyra, A. L. y Pulkkinen, L. (2005). Age of onset of drinking and the use of alcohol in adulthood: a follow-up study from age 8-42 for females and males. Addiction, 100, 652-661

Query, L. R., Rosenberg, H. y Tisak, M. S. (1998). The assessment of young children's expectancies of alcohol versus a control substance. Addiction, 93, 1521-1529

Randolph, K. A., Gerend, M. A. y Miller, B. A. (2006). Measuring alcohol expectancies in youth. Journal of Youth and Adolescence, 33, 939-948.

Rather, B. C., Goldman, M. S., Roehrich, L. y Brannick, M. (1992). Empirical modelling of an alcohol expectancy memory network using multidimensional scaling. Journal of Abnormal Psychology, 101, 174-183.

Smith, G.T. (2008). Comunicación personal.

Spear, L.P. (2000). The adolescent brain and age- related behavioral manifestations. Neuroscience and Biobehavioral Reviews, 24, 417-463.

Velicer, W. F. y Jackson, D. N. (1990). Component analysis versus common factor analysis: some issues in selecting an appropriate procedure. Multivariate Behavioral Research, 25, 1-28

Warner, L. A. y White, H. R. (2003). Longitudinal effects of age at onset and first drinking situations on problem drinking. Substance Use and Misuse, 38, 1983-2016

Wiers, R. W., Sergeant, J. A. y Boudewijn Gunning, W. (2000). The assessment of alcohol expectancies in school children: measurement or modification? Addiction, 95, 737-746

Zamboanga, B. L. (2005). Alcohol expectancies and drinking behaviors in Mexican American college students. Addictive Behaviors, 30, 673-684. 


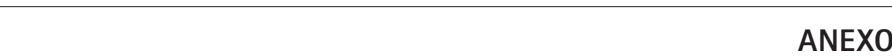

\section{Cuestionario de Expectativas hacia el Alcohol para Niños (CEA-N)}

Para completar el cuestionario, a cada niño se le pide que indique, empleando una escala de cuatro puntos, cuántas veces después de tomar alcohol las personas se sienten del modo que describe cada item. Se utiliza personas como grupo de referencia ya que entre los niños de esta edad la experiencia directa de consumo es baja. Para evitar errores producto de la dispersión o a dificultades de lectura, con los niños de 8 a 10 años la recolección de datos se realiza de manera individual. Especificamente, el entrevistador lee a cada participante las instrucciones y las preguntas, registrando en el cuestionario las respuestas dadas verbalmente por el participante a cada item. Con los niños de 11 y 12 años, si bien la administración es colectiva, las instrucciones y las preguntas son leídas por el investigador, para garantizar la lectura completa de cada ítem.

\section{Puntuación del CEA-N}

El CEA-N está conformado por cinco factores que corresponden a las dimensiones encontradas mediante análisis factorial exploratorio. El puntaje por dimensión se obtiene mediante la suma de las respuestas a cada uno de los ítems. Cada ítem se puntúa con una escala de 1 a 4 , donde $1=$ nunca; $2=$ pocas veces; $3=$ muchas veces y $4=$ siempre. De esta forma, un puntaje elevado implica una mayor anticipación de los efectos de correspondientes a cada escala. La sumatoria del puntaje directo de las tres escalas de EA positivas y de las dos escalas de EA negativas, permite obtener un puntaje general de EA positivas y EA negativas, respectivamente. De esta forma, un mayor puntaje en cualquiera de las escalas, se interpreta como una mayor anticipación de los efectos que describe la escala como consecuencia del consumo de alcohol. Específicamente, un mayor puntaje en la escala sociabilidad es interpretado como una mayor anticipación de una mejora en la interacción social como efecto del consumo.

\begin{tabular}{|c|c|c|c|c|}
\hline El alcohol hace que las personas: & Nunca $=1$ & Pocas veces $=2$ & Muchas veces $=3$ & Siempre $=4$ \\
\hline Hagan cosas que hacen reir a los demás & 1 & 2 & 3 & 4 \\
\hline Piensen que son más que los demás & 1 & 2 & 3 & 4 \\
\hline Se sientan culpables por las cosas que hacen cuando toman & 1 & 2 & 3 & 4 \\
\hline Cuenten chistes & 1 & 2 & 3 & 4 \\
\hline Se sientan superiores al resto & 1 & 2 & 3 & 4 \\
\hline Se sientan más lindas que los demás & 1 & 2 & 3 & 4 \\
\hline Busquen pelearse con otras personas & 1 & 2 & 3 & 4 \\
\hline Se sientan culpables por las cosas que dicen cuando toman & 1 & 2 & 3 & 4 \\
\hline Sean más simpáticas & 1 & 2 & 3 & 4 \\
\hline Se sientan más fuertes que los demás & 1 & 2 & 3 & 4 \\
\hline Digan malas palabras & 1 & 2 & 3 & 4 \\
\hline Sean divertidas & 1 & 2 & 3 & 4 \\
\hline La pasen mejor en lo que estén haciendo & 1 & 2 & 3 & 4 \\
\hline Sean agresivas & 1 & 2 & 3 & 4 \\
\hline Se diviertan más en una fiesta & 1 & 2 & 3 & 4 \\
\hline Lastimen a otras personas & 1 & 2 & 3 & 4 \\
\hline Se sientan tristes & 1 & 2 & 3 & 4 \\
\hline Se rian mucho & 1 & 2 & 3 & 4 \\
\hline Ofendan a las demás personas & 1 & 2 & 3 & 4 \\
\hline Sientan vergüenza por lo que hacen cuando toman & 1 & 2 & 3 & 4 \\
\hline Hablen más & 1 & 2 & 3 & 4 \\
\hline Estén de buen humor & 1 & 2 & 3 & 4 \\
\hline Traten mal a otras personas & 1 & 2 & 3 & 4 \\
\hline Digan cosas de las que después se arrepienten & 1 & 2 & 3 & 4 \\
\hline Se sientan contentas & 1 & 2 & 3 & 4 \\
\hline Se enojen con facilidad & 1 & 2 & 3 & 4 \\
\hline Sean alegres & 1 & 2 & 3 & 4 \\
\hline Se sientan cómodas con lo que están haciendo & 1 & 2 & 3 & 4 \\
\hline Molesten a los demás & 1 & 2 & 3 & 4 \\
\hline Discutan con los demás & 1 & 2 & 3 & 4 \\
\hline
\end{tabular}


